\title{
Formación en Valores Éticos: ¿Posibilidad o Utopía?
}

\section{Formation of values: ¿possibility or a utopian dream?}

\begin{abstract}
Alexander González García
Magister en investigación y Magister en Gestión Educativa de la Universidad Saint Joseph de Beirut, Líbano; Candidato a doctor en Ciencias de la Educación de la Universidad Nacional de la Plata, Argentina. Filósofo de la Pontificia Universidad Javeriana de Bogotá, Colombia; Teólogo de la Universidad de Londres, Inglaterra; Especialista en Islam y Estudios Árabes del Instituto Pontificio Dar Comboni de El Cairo, Egipto. Docente de la Universidad del Norte, Barranquilla, Colombia, Docente de Humanidades y Filosofía, Email: alexandersj@hotmail.com
\end{abstract}

Para citar este artículo: González A., (2016). Formación en Valores Éticos: ¿Posibilidad o Utopía?. Escenarios, 14 (2), p,p 120-128 DOI: http:/ / dx.doi.org/10.15665/ esc.v14i2.936

Recibido: agosto 18 de 2016

Aprobado: octubre 3 de 2016

\begin{abstract}
RESUMEN
Frente a los problemas sociales y los desafíos de convivencia de las sociedades contemporáneas, nos preguntamos si ¿la formación en valores es posible, o es una utopía humana? En el marco de una investigación doctoral titulada "Formación en valores éticos en estudiantes de pregrado" que se desarrolla bajo la dirección de la Universidad Nacional de la Plata, Argentina se ha venido estudiando esta pregunta desde un enfoque filosófico y pragmático. En este artículo, desde una mirada filosófica, examinamos los principales conceptos de este tipo de formación como son "valor", "ser humano" y "formación". Luego, desde un enfoque más pragmático, se deriva al caso de la educación superior para mirar en qué medida la formación en valores es un hecho o una simple idea. Al nivel filosófico, se concluye que la formación en valores es una necesidad humana de transcender de un comportamiento animal a un comportamiento humano. Esto es debido a que los valores éticos son concebidos como objetivos y universales y por ello la formación se da a través de la construcción de la personalidad moral de cada persona. En el caso de la educación superior, ésta formación es posible cuando la institución educativa asume verdaderamente su misión de formar individuos para el progreso de la sociedad y esto incluye la apropiación de valores éticos. Solo cuando esto se da, la formación en valores en la educación superior es una realidad y no una simple utopía.
\end{abstract}

Palabras clave: Formación en valores éticos, valor, ser humano, formación, educación superior. 


\section{ABSTRACT}

In the face of social problems and other challenges confronting contemporary societies, we ask whether the formation of values, as such, is possible or just a utopian dream? As a part of a doctoral research titled "Formation in ethical values in undergraduate students" under the direction of Universidad Nacional de la Plata, Argentine, we explore this question in reference to both a philosophical and pragmatic perspective. In this article, from a philosophical perspective, we shall, firstly, examine the main concepts "value", "human being", and "formation". Secondly, from a pragmatic view point we look concretely at the case of higher education with a view to determine whether the formation of values exist as a given reality or, indeed, remains only as an ideal. From the philosophical perspective we conclude that the formation of values relates to the human need to transcend from animal behaviour to human behaviour. This is because ethical values are understood as both objective and universal, and formation in them comes through the construction of an individual moral personality of a person. This formation is possible in the context of higher education when the educational institution assumes explicit responsibility for forming people for the progress of society, a process that includes the appropriation of ethical values. Only when this happens does formation in values in higher education prove to be a reality and not a utopian dream.

Key words: Formation of values, value, human being, formation, higher education.

\section{INTRODUCCIÓN}

Frente a los problemas sociales y los desafíos de convivencia de las sociedades contemporáneas, la necesidad de formar en valores tiene cada vez más sentido. Esto se debe a que la función de los valores en la sociedad es de vital magnitud, pues permiten el buen funcionamiento de la colectividad, mantienen la identidad personal y general de una sociedad y, por lo tanto, conlleva a la cohesión entre sus miembros.

Sin embargo, en la actualidad los valores éticos se están dejando a un lado y se reemplazan por otro tipo de valores que conllevan a desestabilizar la convivencia entre los ciudadanos. En las sociedades modernas, probablemente por la globalización, se observan los valores éticos como una realidad subjetiva de los individuos y, en consecuencia, se les considera más relativos que absolutos.

Frente a este relativismo y a los problemas sociales que esto conlleva, la Declaración de los Derechos Humanos, proclamada por la ONU en 1948, pone de relieve la dignidad humana bajo la luz de valores éticos, y sigue siendo una orientación válida para el mundo contemporáneo. Esta declaración busca ser una guía para el desarrollo y el bienestar individual y colectivo de los ciudadanos en las sociedades actuales. Sin embargo, estos derechos sólo se hacen posibles cuando los ciudadanos incorporan en sí mismos los valores de igualdad, libertad, tolerancia, respeto, justicia, solidaridad, etc.

Los valores éticos encierran los comportamientos deseables para formar a las nuevas generaciones y de ellos depende la armonía de la sociedad. En palabras de Adela Cortina, los individuos y las sociedades que viven bajo valores éticos tienen "agallas" para afrontar la vida con "altura humana" (1995, p. 48). De ahí que sea necesario revitalizar el compromiso social de las instituciones sociales. Los centros de educación básica, media y superior tiene el rol fundamental de reactivar el compromiso social para el desarrollo de la sociedad. Sin embargo, por la realidad humana que vivimos ¿la formación en valores es posible, o es una utopía?

En el marco de una tesis doctoral en curso titulada "Formación en valores éticos en estudiantes de pregrado" que se desarrolla bajo la dirección de la Universidad Nacional de la Plata, Argentina; se ha estado respondiendo a esta pregunta, desde una mirada filosófica, profundizando en los principales conceptos que encierra la formación en valores. Debido a que la axiología y la antropología están estrechamente vinculadas nos aproximaremos a los conceptos de "valor", 
de "ser humano" y de "formación". Después, desde un enfoque más pragmático, estudiaremos en qué medida la formación en valores es una realidad o una utopía en la educación superior. Iniciemos nuestro estudio con la clarificación conceptual.

\section{Aproximación a la definición de valor.}

Para poder realizar una definición apropiada del valor es necesario primero abordar su naturaleza. Frente a esto, algunos filósofos como Ralph Barton Perry (1876-1957), Bertrand Russell (1872-1970) y Jean Paul Sartre (1905-1980) afirman que el valor es subjetivo, pues carece de contenido de manera independiente del sujeto que los experimenta. Para otros, como Max Sheler (1874-1928), Nicolari Hartmann (1882-1950) y José Ortega y Gasset (1883-1955) junto a autores más actuales como Octavi Fullat (1928) el valor trasciende de la experiencia individual de un sujeto y, por lo tanto, es objetivo e independiente de una realidad particular.

La mirada subjetivista es adecuada en cuanto el valor tiene un relación estrecha con las valoraciones humanas, pues “¿qué sentido tendría la existencia de valores que escaparan a toda posibilidad de ser apreciados por el hombre?" (Frondizi, 1972, p. 20). Sin embargo, la postura objetivista también tiene un aporte válido en esta reflexión, ya que aunque la valoración es completamente humana, el valor es independiente de las valoraciones humanas (juicios de valor personales). Es decir, "si no hubiera valores ¿qué habríamos de valorar?" (Frondizi, 1972, p. 21).

Para los subjetivistas el valor se fundamenta en el deseo del sujeto, pues el deseo hace que el objeto tenga valor. Si llegase el caso de que el sujeto pierde el deseo por el objeto, de inmediato el valor concedido a éste también se pierde. Por el contrario, para los objetivistas el valor no se reduce al deseo, pues existe también la valoración a cosas que no deseamos. Por ejemplo, la acción de ayudar a nuestro enemigo que está en dificultades es un acto valioso del deber moral que va más allá de nuestros intereses o deseos personales. Frente a esto, estamos encerrados en el juego dialéctico de saber si las cosas poseen un valor en sí mismas o será nuestro interés el que coloca el valor en ellas. Desde este enfoque, la discusión para saber la naturaleza del valor es interminable.

Risieri Frondizi (1972) propone una postura intermedia para analizar esta problemática. Para este filósofo, todos los valores no son iguales y pertenecen a diversas jerarquías en la escala axiológica. Hay valores más "bajos" como los que se refieren a algo agradable o desagradable y otros más "altos" como los que se relacionan con el comportamiento ético. Los valores "bajos" dependen más de la subjetividad del individuo, pues es él quien dice qué le gusta y qué no le gusta. Sin embargo, en los valores más "altos" la valoración ética no puede corresponder a la subjetividad, pues el valor ético contiene en sí una fuerza impositiva que es independiente de los deseos personales.

La interpretación de Frondizi ofrece luces para la asimilación del valor en las sociedades contemporáneas, pues el riesgo actual es de considerar los valores éticos como una simple opción personal. La distinción entre los valores "bajos" y los valores "altos" permite poner de relieve la naturaleza objetiva de los valores éticos y la no dependencia de los deseos particulares de un individuo. En consecuencia, los valores éticos entran en la categoría del deber ser de una sociedad.

De lo anterior, también podemos deducir la importancia de unos valores frente a otros. Aunque es más fácil afirmar la existencia de una jerarquía de valores que ofrecer un escalonamiento de los mismos. Esto, en virtud de la importancia de un valor frente a otros, que depende del momento histórico que una sociedad vive en particular. A pesar de los intentos por elaborar o crear una tabla jerarquizada de valores, como la tabla axiológica de Max Scheler (1913/2001), estos intentos no ofrecen de manera suficiente una cohesión y solidez argumentativa, o lógica, pues los valores son fluctuantes y dependen de la realidad humana.

Además de la característica de la jerarquía de los valores, podemos añadir que éstos son polarizados, es decir, existen valores positivos y a su vez existen sus contrarios, los valores negativos. Sin embargo, la existencia de unos no depende de la ausencia de los otros. Por ejemplo, un acto injus- 


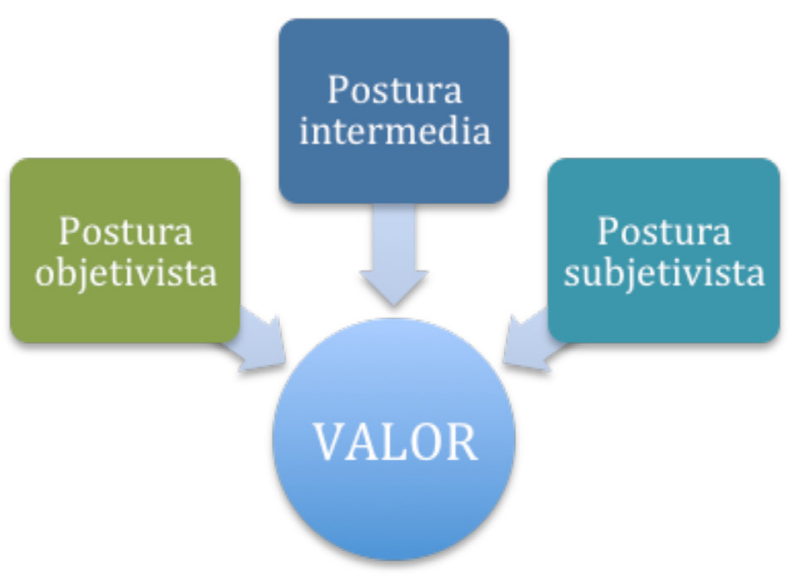

Fuente: Elaboración propia

to se puede originar sin que tenga una relación con la justicia. Como consecuencia de esta polaridad, el ser humano no puede ser indiferente en el mundo, pues frente a los hechos no puede quedarse indiferente, asume un valor -positivo o negativo- y junto a ese valor asume una postura que define su tipo de relación con esa realidad particular. Frondizi explica esto diciendo que no hay "persona que se mantenga indiferente al escuchar una sinfonía, leer un poema o ver un cuadro" (1972, p.14).

Entonces, los valores son cualidades de las cosas o de las personas, son polarizados, pues cada valor tiene su contrario, son jerarquizados, pues hay unos más importantes que otros y son objetivos cuando se trata específicamente de valores éticos. Pero, valorar un objeto, una acción, o una persona es el resultado de un proceso subjetivo.

Para la asimilación de valores éticos por parte de los individuos es necesaria la formación en ellos, pues el ser humano construye su mirada del mundo a través de los principios que adquiere a lo largo de su vida. Por eso es importante estudiar quién es el ser humano para ser formado en valores.

\section{Aproximación a la definición del ser humano.}

A lo largo de la historia, han existido diversos enfoques que han intentado definir al ser humano. Muchas definiciones han tomado como referencia la dimensión biológica del hombre y lo han definido como ser político (Aristóteles), ser proletario (Marx), ser simbólico (Ernst Cassirer), ser cultural (Tomas de Aquino), etc.. Sin embargo, estas definiciones abordan solamen- te algunos aspectos humanos. De ahí que muchos filósofos reflexionan sobre la definición del hombre sin reducirlo a una sola dimensión de la realidad humana (Ferry \& Vincent, 2001). En esta línea, Paul Ricouer presenta principalmente en su obra Finitud y Culpabilidad (1991) un análisis sobre la naturaleza del hombre y su búsqueda de valores, pertinente para nuestro análisis.

En el ser humano existen dos realidades, una biológica o material y otra espiritual o no material. Estas dos dimensiones están estrechamente relacionadas y conviven en una total interdependencia. La parte material o biológica del ser humano permite que se sitúe concretamente en un medio determinado bajo la influencia de su entorno, y es en ese medio donde él manifiesta los impulsos y los deseos a través de sus acciones voluntarias o involuntarias. Las acciones humanas son influenciadas por el ambiente, la cultura, los valores sociales, la educación y la historia personal y social.

Por otro lado, en el ser humano co-existe también la dimensión espiritual que lo hace trascender de su corporeidad. Ante la influencia del ambiente donde se encuentre, el ser humano puede aceptar o no las dinámicas de la sociedad, así como también puede asumir un rol activo para transformar su presente y el de la comunidad.

En las acciones del hombre participan la dimensión corporal y la dimensión espiritual, y es esta segunda la que le permite ir más allá de un comportamiento animal a un comportamiento humano. Sin embargo, esta posibilidad vive en la constante tensión entre buscarse a sí mismo o transcender de su realidad hacia los otros. En otras palabras, aunque el comportamiento humano es fruto de su libertad, este no es completamente autónomo pues está condicionado por su ser espiritual y su ser material; así pues, la voluntad humana vive en un doble movimiento: "de una parte es indeterminada y, de otra, es determinada; es acción y pasión; conciencia e inconsciencia" (Kagelmacher, 2011).

Frente a esta realidad, Ricoeur desarrolla el concepto de labilidad entendida como la conciencia de los límites propios del hombre. En palabras de Descartes: "me veo expuesto a una infinidad de fallos así que no debo extrañarme de mis des- 
lices" (IVa Meditación, citado por Ricoeur 1991, p. 125). Por un lado, el hombre aspira y espera lo que desea, pero en la realidad no todo lo logra, y por eso en la realidad humana "toda potencia tiene el reverso de toda impotencia" (Ricoeur, 1991, p. 103).

Esta realidad finita del hombre se ve reflejada en la propia corporeidad de su ser que ofrece la oportunidad de interactuar con el mundo, pero de una manera limitada. Sin embargo, esta finitud impulsa al ser humano al deseo y "el deseo nos transporta fuera de nosotros; nos sitúa ante lo deseable, que a su vez está en el mundo" (Ricoeur, 1991, p. 100). Entonces, el deseo impulsa al propio hombre a anhelar algo mejor, y esto va más allá de los objetos sensibles y "trasciende" a los valores. Sus anhelos lo pueden conducir a mirarse de manera egoísta o de manera altruista, pero es esta segunda posibilidad la que permite que el hombre viva una "vida auténtica, coherente y abierta a los demás" (Kagelmacher, 2011). De esta manera, el ser humano es accesible "a todos los valores de todos los humanos a través de todas las culturas" (Ricoeur, 1991, p. 108), pero él accede a los valores de manera individual, a partir de su carácter, historia, cultura, educación, sociedad, etc. De ahí, se forma la "personalidad moral", que es el resultado de la apropiación de todos esos factores externos y que integran su manera de proceder en el mundo (Rovira, 1996).

El ser humano es un ser complejo que está en constante apertura hacia el mundo y en esa dinámica es consciente de su ser finito y de su anhelo de infinitud; pero para trascender sus propios límites, los valores éticos se presentan como la posibilidad de realización de este deseo. La manera de acercarlo a estos valores es por medio de la formación.

\section{Aproximación a la definición de formación.}

Como en el ser humano no existe un destino escrito a priori, pues es un ser abierto al mundo en constante transformación y continuo desarrollo, el ser humano tiene siempre la posibilidad de crecer, de aprender, de progresar, de perfeccionarse, y esta labor nunca está acabada. En este transcurso, la formación juega un rol fundamental, pues como el hombre es un ser inacabado necesita de la formación para construirse a sí mismo y auto realizarse.

A propósito de esto, Heidegger afirma que el ser humano necesita "meditar" y "cuidarse" para que sea completamente "humano" y no "inhumano" (1970, p. 3). Entonces, el papel de la formación es el de construir al hombre alejándolo de su comportamiento animal para realizarlo como sujeto que participa, junto con otros, en una sociedad. Por lo tanto, la formación busca develar y potencializar todas las capacidades y posibilidades del ser humano.

En este sentido, cada persona busca su perfeccionamiento individual y, en la medida de su estado formativo, también desea y actúa en el mejoramiento de su cultura (Villegas, 2011; Turcan, Reilly, \& Bugaian, 2016). Por eso, la formación pone de relieve los valores éticos como medios para potencializar lo humano en cada cultura, ya que este proceso nunca es un acto individual y egoísta, sino que se da en una realidad determinada para el desarrollo del sujeto y de su contexto social. Esto significa que el ser humano siempre está en constante evolución y, en consecuencia, siempre está en continua formación.

Sin embargo, la gran dificultad actual es reducir el fecundo sentido que tiene el concepto de formación a una simple instrucción didáctica. Esto se debe a una vinculación del término formación con un objetivo específico a realizar, o como dice Villegas se trata "de formar 'para' algo o 'en algo'" lo cual conlleva a una simple "formación en actos" (2011, p. 9). Aunque la formación implica una experiencia humana, no se reduce a unos actos educativos realizados en una época específica de la vida. La formación no se agota en la obtención de un título universitario sino que es una función propia del ser humano, pues "se trata de una función evolutiva que se ejerce según un cierto proceso y desencadena la experiencia constitutiva del ser" (Villegas, 2011, p. $11)$.

Ahora bien, a lo largo de la historia existen diversas posturas y visiones sobre la comprensión de la formación en valores. Por un lado, encontramos varios autores que interpretan la formación en valores como un medio para inducir al sujeto a vivir en sociedad y, por eso él -el ser humano-, debe asimilar los valores del grupo social 
al que pertenece (Durkheim, 2002; Ruíz \& Vallejos, 2003; López, 2005). Esta dinámica conduce a una incorporación de valores que no son propios del individuo. En este modelo, la postura formativa del sujeto es completamente pasiva, pues se limita a recibir valores sin desarrollar una verdadera autonomía ética. Sin embargo, el ser humano que está siempre en cambio y, en consecuencia, en constante formación, no puede asumir valores sin tener una postura crítica.

Por otro lado, frente a la discusión sobre la comprensión del "valor" como cualidad objetiva u subjetiva, existe un paradigma de formación que apoya más la mirada subjetivista. En esta óptica, los valores son relativos y la formación en ellos dependen sólo de la libertad del individuo que los esté deseando asimilar (Reyero, 2001). Esta visión reduce el papel de la formación ética a un simple desarrollo de la capacidad de discernimiento de los valores de la persona. Aunque este tipo de formación implica cierto grado de autonomía, también es completamente individualista. No obstante, el ser humano es un sujeto social que se construye en comunidad, $y$, en efecto, su escala de valores personal no puede ser completamente abstracta del contexto donde se encuentra.

Frente a los límites de éstas y otras posturas, Rovira (1995) propone concebir la formación en valores como una "construcción de la personalidad moral" (p. 110). Para este investigador, los valores éticos no se descubren, por el contrario, se construyen de manera personal y esta elaboración es el fruto de la interacción individual con el medio y con la sociedad. Es decir, el proceso de construcción de valores personales implica un reconocimiento de los valores culturales.

Por eso, según Rovira, la construcción de la personalidad moral contiene un desarrollo adaptativo al contexto y a sí mismo. Este proceso consiste en la asimilación de "pautas sociales básicas de convivencia" y del "reconocimiento" de deseos, posturas, posiciones y creencias que se valoran personalmente (Rovira, 1995, p.111). A pesar de que estos dos procesos de adaptación son muy importantes, su integración no puede ser el objetivo último de la formación en valores, pues quizás existe una serie de valores como la justicia, la libertad, la igualdad y la solidaridad

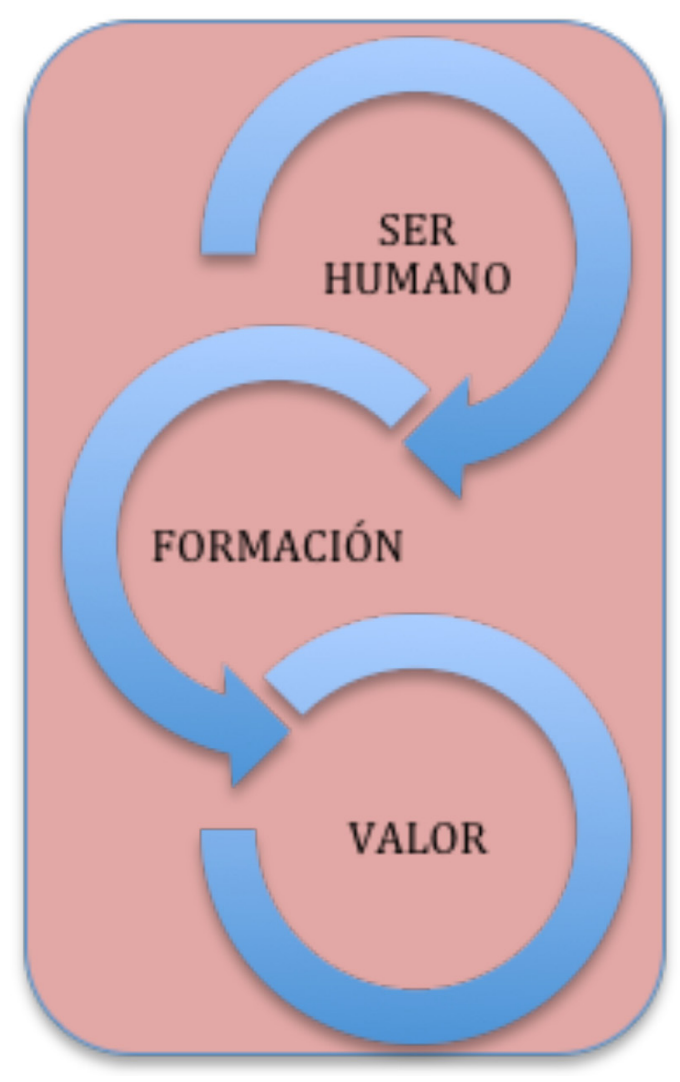

Fuente: Elaboración propia

que no están presentes ni en la sociedad, ni en el sujeto, pero que son "horizontes normativos deseables" (Rovira, 1995, p. 112). Por esta razón, es necesario formar la autonomía moral del sujeto a través del desarrollo del juicio, la comprensión y la autorregulación para que sea capaz de afrontar con carácter los conflictos de valores que viven las sociedades contemporáneas. Entonces, la formación en valores consiste en la educación de la "conciencia moral autónoma de cada sujeto" y este es un proceso de construcción individual que tiene como finalidad última el desarrollo de la persona y la armonía de la sociedad.

Hemos visto hasta el momento que la formación en valores, en el espacio de la filosofía, es una necesidad humana y, por lo tanto, una posibilidad. Pero, en el contexto de lo pragmático debemos averiguar si es todavía viable o en cambio es una utopía. Para esto vamos a tomar como referencia la universidad, pues, por las interacciones que en ella surgen, podría ser un espacio privilegiado para dar las bases de una sólida formación humana. 


\section{La universidad como espacio para la formación en valores}

La universidad es metafóricamente conocida como la "Alma Mater", es decir, como una madre que proveé de alimento intelectual a cada uno de sus hijos; de ahí que sea considerada como una institución con ciertas características especiales. Varios autores afirman que la singularidad de la universidad es la de llegar a ser una comunidad ética (Bara \& Martínez, 2012; Newman, 1999; Esteban, Buxarrais, \& Martínez, 2002).

La etimología de la palabra universidad nos remite a otros conceptos como corporación o comunidad. Esto se entiende en la práctica, pues existe, por ejemplo, la relación entre profesores y estudiantes que se reúnen en "común-unión" alrededor del conocimiento. Sin embargo, la universidad no es cualquier tipo de asociación, pues su fin de indagar por el saber la hace diferente (Bara \& Martínez, 2012), y en esa búsqueda la ética hace parte esencial de su definición.

Esto se debe, en primer lugar, a que la universidad busca transmitir e influenciar a sus estudiantes en esa búsqueda de conocimiento, y este proceso, ciertamente, trae consigo connotaciones éticas. La relación profesor - estudiante es fundamental para que el aprendizaje sea fructífero, y esta relación humana exige un comportamiento ético y un bienestar psicológico. Así, "la comunidad universitaria se convierte en un conjunto de maestros y estudiantes obsesionados por la Verdad, la Belleza y el Bien, y por lo tanto, se convierte en un grupo de personas que conforman una comunidad ética" (Bara \& Martínez, 2012, p. 82).

Además de esto, la universidad es el lugar donde se busca la excelencia profesional, de ahí que la universidad se ocupe del tipo de profesional que quiere ofrecer a la sociedad. Esto no se reduce solamente al conocimiento de la ética profesional, incluye también la ética del profesional y, por lo tanto, el objetivo de la formación universitaria implica el crecimiento deontológico y ético del futuro profesional (Esteban, Buxarrais, \& Martínez, 2002; Olmeda, Martín, \& Uceda, 2011).

Así mismo, la universidad es una comunidad ética debido al compromiso que ella tiene con el contexto social. A lo largo de la historia de la educación superior vemos la estrecha relación entre la sociedad y la universidad, pues ésta última ha buscado formar ciudadanos que influyan en la realidad que les rodea.

En efecto, la formación universitaria tiene como una de sus prioridades mejorar la sociedad y esta apuesta no le permite mantenerse al margen. Por eso, en la actualidad, se habla de una responsabilidad social universitaria que implica, por una lado, brindar una formación sólida de profesionales para el mejoramiento de la comunidad y, por otro, ofrecer investigaciones que pretendan contribuir en el avance de la sociedad. Este compromiso social no es el resultado de una acción "filantrópica", es un modo permanente de operar la universidad, que tiene implicaciones éticas ad-intra y ad-extra del establecimiento educativo (Vallaeys, 2014).

Entonces el Alma Mater no puede reducirse, por ningún motivo, a una comercialización de títulos universitarios donde lo importante sería sólo la formación intelectual del estudiante. Para que una universidad sea universidad debe ser coherente con su razón de ser, esto implica también ofrecer una formación ética al estudiante para que pueda afrontar y contribuir, de manera idónea, a los desafíos de la sociedad. En otras palabras, la formación universitaria no se limita a un progreso individual del sujeto, implica un desarrollo de sus capacidades en pro de la sociedad.

Para los pedagogos, la formación en valores tiene que ver con "compromisos valorativos fundamentales" y esto es fruto de cómo el valor hace parte de la existencia motivacional del individuo. No se trata de un conocimiento racional del valor, pues, por ejemplo, la mayoría de los ciudadanos sabe la importancia del respeto dentro de una sociedad y no por eso todos lo practican.

El valor es incorporado por un individuo o por una sociedad en la medida que sienten la necesidad de proceder de acuerdo con ese valor. Entonces, "sólo cuando los valores constituyen motivos de la actuación del sujeto se convierten en verdaderos reguladores de su conducta" (Maura, 2001, p. 17). Esta "construcción de la personalidad moral" del sujeto, se da como resultado de 
un largo proceso educativo en el cual participan la familia, la sociedad y las instituciones educativas.

En el caso de los estudiantes universitarios de pregrado, la educación en valores es de vital importancia, porque es en esta época cuando los jóvenes consolidan "el sistema motivacional y cognitivo" que va a guiar su manera de proceder en el mundo (Maura, 2001, p. 21).

Para ello, la universidad debe tener en cuenta que el proceso de aprendizaje no se limita a una formación académica, ni a un desarrollo técnico del estudiante; sino que incluye el desarrollo emocional y motivacional de la persona. El clima organizacional de la universidad si es vivido de manera ética, genera un ambiente favorable para la vivencia de valores éticos por parte de todos los miembros de la institución, lo cual presenta al estudiante la importancia de los valores éticos y la necesidad de ellos para la vivencia en armonía.

Además, la educación en valores se concretiza en la visión humanística de los currículos universitarios, sobre todo de pregrado, que permiten al estudiante reflexionar sobre lo humano y comprender los debates actuales de las sociedades contemporáneas.

Por otra parte, el docente también juega un papel fundamental en la formación en valores, pues en la medida que propicia y acompaña al estudiante en la reflexión sobre su comportamiento, este último avanza en la auto-construcción de valores éticos. Pero para que esto sea genuino, el docente primero debe actuar bajo valores éticos.

Como hemos visto, desde la filosofía, la formación en valores es una necesidad humana de transcender de un comportamiento animal a un comportamiento humano. Para ello, los valores éticos son concebidos como objetivos y universales y la formación en ellos se da a través de la construcción de la personalidad moral de cada individuo. En la universidad, esta formación es posible cuando la institución educativa asume verdaderamente su misión de formar individuos para el progreso de la sociedad y esto incluye la apropiación de valores éticos. Sólo cuando el clima institucional, la pedagogía utilizada, los currículos de las carreras y los docentes buscan educar bajo criterios éticos a sus estudiantes, la formación en valores en la universidad deja de ser una utopía para ser una realidad.

\section{Referencias}

Bara, F. E., \& Martínez, M. (2012). ¿Son universidades todas las universidades? La universidad como comunidad ética. Bordón. Revista de pedagogía, 64(3), 77-92.

Cortina, A. (1995). La educación del hombre y del ciudadano. Revista Iberoamericana de educación, (7), 41-64.

Durkheim, É. (2002). La educación moral. Ediciones Morata.

Esteban, F. E., Buxarrais, M. R. B., \& Martínez, M. M. (2002). La universidad como espacio de aprendizaje ético. Revista Iberoamericana de educación, (29), 17-44.

Ferry, L., \& Vincent, J.-D. (2001). ¿Qué Es el Hombre?: Sobre Los Fundamentos de la Biología y la Filosofía. Taurus.

Frondizi, R. (1972). Qué son los valores?: introducción a la axiología. Recuperado a partir de http: / / www.sidalc.net/ cgi-bin/ wxis.exe/ ?IsisScript=AGRIUAN.xis\&method=post\&formato $=2 \&$ cantidad $=1 \&$ expresion $=\mathrm{mfn}=000166$

Gasset, J. O. y. (2010). Introducción a una estimativa. ¿Qué son los valores? Encuentro.

Heidegger, M. (1970). Carta sobre el humanismo. Taurus. Recuperado a partir de http://www. academia.edu/download/32473227/CSH.pdf

Kagelmacher, M. (2011). Los valores educativos en la postmodernidad: una propuesta desde la filosofía de la educación de Octavi Fullat. Recuperado a partir de http:/ / hdl.handle. net/10803/32194

López, H. F. (2005). Pautas de transmisión de valores en el ámbito familiar. Recuperado a partir de https:/ / digitum.um.es/xmlui/handle/10201/9703

Maura, V. G. (2001). La educación de valores en el currículum universitario. Un enfoque psicopedagógico para su estudio. Pedagogía Universitaria, 6(1). Recuperado a partir de http:/ / cvi. 
mes.edu.cu / peduniv / index.php / peduniv / article/download/168/164

Newman, J. H. C. (1999). The idea of a university: Defined and illustrated. Regnery Publishing. Recuperado a partir de https://books.google. com / books?hl=es\&lr=\&id=kadoNcxRXyQ$\mathrm{C} \&$ oi $=$ fnd \&pg $=$ PR9\&dq $=$ the + idea + of $+a+$ university\&ots=Ak1rxqfaSm\&sig=cg8xgBVHgBiOv0alzA-oxmMcwuw

Olmeda, G. J., Martín, E. L., \& Uceda, P. Q. (2011). La universidad como espacio cívico: valoración estudiantil de las modalidades de participación política universitaria. Revista de educación, (1), 69-91.

Reyero, D. R. (2001). El valor educativo de las identidades colectivas: cultura y nación en la formación del individuo. Revista española de pedagogía, 105-120.

Ricoeur, P. (1991). Finitud y culpabilidad, trad. de Alfonso García y Luis M. Valdés. Madrid: Taurus.

Rovira, J. M. P. (1995). Construcción dialógica de la personalidad moral. Revista iberoamericana de educación, (8), 103-120.

Rovira, J. M. P. (1996). La construcción de la personalidad moral. Grupo Planeta (GBS).

Ruíz, P. O., \& Vallejos, R. M. (2003). FAMILIA Y TRANSMISIÓN DE VALORES. Revista interuniversitaria, 33-53.

Scheler, M. (2001). Ética: nuevo ensayo de fundamentación de un personalismo ético. Caparrós editores.

Turcan, R. V., Reilly, J. E., \& Bugaian, L. (Eds.). (2016). (Re)Discovering University Autonomy. New York: Palgrave Macmillan US. Recuperado a partir de http://link.springer. com $/ 10.1057 / 9781137388728$

Vallaeys, F. (2014). La responsabilidad social universitaria: un nuevo modelo universitario contra la mercantilización. Revista iberoamericana de educación superior, 5(12), 105-117.

Villegas Durán, L. A. (2011). Formación: apuntes para su comprensión en la docencia universitaria. Recuperado a partir de http:/ / digibug.ugr. es/handle/10481/15185. 